\title{
DIVERSIDADE ESTRUTURAL EM Eugenia L. (MYRTACEAE)
}

\author{
STRUCTURAL DIVERSITY IN Eugenia L. (Myrtaceae)
}

\author{
Sidinei Rodrigues dos Santos ${ }^{1}$ José Newton Cardoso Marchiori ${ }^{2}$ Anelise Marta Siegloch ${ }^{3}$
}

\begin{abstract}
RESUMO
No presente estudo é investigada a anatomia da madeira de 9 espécies sul-rio-grandenses de Eugenia (Myrtaceae), com vistas ao reconhecimento de caracteres úteis à identificação do gênero e espécies. Os resultados demonstram uma grande homogeneidade estrutural, fruto do elevado número de caracteres anatômicos compartilhados. Nenhuma característica ocorre exclusivamente no grupo taxonômico em questão. É confirmado o valor do arranjo do parênquima axial para a separação de espécies, bem como da frequência de poros e características de raios. Não é possível contestar, com base na anatomia da madeira, a inclusão de Hexachlamys em Eugenia, como sugerido por Landrum e Kawasaki (1997).
\end{abstract}

Palavras-chave: Anatomia; taxonomia; madeira; Myrteae.

\begin{abstract}
The wood anatomy of nine species of Eugenia (Myrtaceae) native in Rio Grande do Sul State (Brazil) is presently studied in order to identify diagnostic characters useful to identify genus and species. A great structural homogeneity was observed, reflecting the high number of shared anatomical features. None characteristic is exclusive to this taxonomic group. The value of axial parenchyma arrangement to species segregation is confirmed, as well as the frequency of pores and characteristics of rays. It is not possible to disprove, based on wood anatomy, the inclusion of Hexachlamys in Eugenia, as suggested by Landrum and Kawasaki (1997).
\end{abstract}

Keywords: Wood; taxonomy; anatomy; Myrteae.

\section{INTRODUÇÃO}

Com cerca de 1000 espécies distribuídas principalmente nas Américas Central e do Sul (MERWE et al., 2005), o gênero Eugenia é, ao lado de Myrcia, um dos mais representativos da família Myrtaceae, inclusive no Brasil, onde são encontradas aproximadamente 350 espécies nativas de Eugenia (LANDRUM e KAWASAKI, 1997). Trata-se, também, de um dos grupos mais importantes em diversidade e abundância de espécies lenhosas do
Rio Grande do Sul (KLEIN, 1984), que, de acordo com Sobral (2003), contabiliza 31 espécies.

Além da importância ecológica, as espécies do gênero prestam-se a diversos usos, com destaque para a produção de frutos, madeira e paisagismo (RECORD e HESS, 1949; MARCHIORI e SOBRAL, 1997; OLIVEIRA, 2007).

A circunscrição de Eugenia, assim como da família Myrtaceae como um todo, tem experimentado importantes modificações nos últimos anos. Landrum e Kawasaki (1997), em

1 Biólogo. Dr., Universidade Federal do Pampa, Rua 21 de Abril, 80, Bairro São Gregório, CEP 96450-000, Dom Pedrito (RS), Brasil. sthurt.bio@gmail.com

2 Engenheiro Florestal, Dr., Professor Titular do Departamento de Ciências Florestais, Ciências Florestais, Centro de Ciências Rurais, Universidade Federal de Santa Maria, Av. Roraima, 1000, CEP 97105-900, Santa Maria (RS), Brasil.marchiori@pq.cnpq.br

3 Engenheira Florestal, Doutoranda, Programa de Pós-Graduação em Engenharia Florestal, Departamento de Ciências Florestais, Centro de Ciências Rurais, Universidade Federal de Santa Maria, Av. Roraima, 1000, CEP 97105-900, Santa Maria (RS), Brasil. anesiegloch@yahoo.com.br 
revisão das espécies brasileiras da família, reduziram à sinonímia deste gênero todos os representantes de Hexachlamys e Calycorectes, por serem ambíguas ou de difícil observação as diferenças que os mantinham separados em grupos taxonômicos distintos. Tal interpretação, ainda não completamente consolidada, aguarda, todavia, confirmação por mais estudos.

Neste contexto, a Anatomia da Madeira ocupa um papel de destaque, pois, além de servir para a identificação de espécies, gêneros e famílias botânicas, o método anatômico é empregado, com sucesso, em investigações taxonômicas, filogenéticas e evolutivas, entre outros estudos (METCALFE e CHALK, 1983).

Apesar da importância, são ainda escassas as referências sobre a composição estrutural das madeiras de Eugenia, especialmente no Brasil, onde ocorre um grande número de espécies. Mainieri e Chimelo (1989) fazem uma breve caracterização da estrutura anatômica de uma espécie não identificada do gênero em questão. Cury (2001) descreveu a anatomia da madeira de Eugenia uniflora, com base em coleta realizada na Mata Atlântica de São Paulo. Barros et al. (2001, 2003) fornecem a descrição microscópica detalhada de cinco espécies de Eugenia e outras Myrtaceae nativas no Rio de Janeiro. Marques et al. (2007), por sua vez, investigaram a estrutura anatômica de três espécies de Eugenia provenientes de remanescentes florestais do mesmo Estado.

Trabalhos de cunho taxonômico envolvendo espécies de Eugenia são ainda mais raros na literatura. Détienne e Jacquet (1983) estudaram a anatomia da madeira de alguns representantes de Eugenia e de outros 7 gêneros da Amazônia brasileira, com vistas à identificação, e Soffiatti e Angyalossy-Alfonso (1999) realizaram um estudo comparativo do lenho e casca de duas espécies de Eugenia da Mata Atlântica de São Paulo, com o mesmo objetivo. Em ambos os casos observou-se pouca variabilidade estrutural, sendo o parênquima axial o caráter anatômico de maior importância para a separação das espécies. Rebollar-Domínguez e Tapia-Torres (2010), por sua vez, investigando a anatomia da madeira de duas espécies do gênero em questão, nativas no México, salientaram para o seu reconhecimento, além do arranjo do parênquima axial, a largura de raios e a presença ou não de conteúdos em fibras. Na literatura sobre o gênero, destacam-se, ainda, os trabalhos de Record e Hess (1949) e Metcalfe e Chalk (1972). Neles, os autores fornecem uma caracterização geral da família, com alguns comentários de cunho taxonômico.

No presente estudo pretende-se investigar a anatomia da madeira de 9 espécies de Eugenia nativas no Rio Grande do Sul, com vistas ao reconhecimento de caracteres úteis à identificação do gênero e espécies, e ao esclarecimento das questões taxonômicas do grupo.

\section{MATERIAL E MÉTODO}

Ao todo, foram estudadas 9 espécies sul-

TABELA 1: Material investigado.

TABLE 1: Investigated material.

\begin{tabular}{lccc}
\hline \multicolumn{1}{c}{ Espécie } & Amostra $^{1}$ & Local de coleta (RS) & Referência \\
\hline Eugenia burkartiana (D. Legrand) D. Legrand & 2812 & Tenente Portela & Santos e Marchiori, 2010 \\
Eugenia hiemalis Cambess. & 5904 & Restinga Seca & Santos e Marchiori, 2011b \\
Eugenia involucrata DC. & 662 & Mata & Marchiori, 1984 \\
& 2387 & São Vicente do Sul & Marchiori e Santos, 2009 \\
Eugenia mansoi O. Berg & 2391 & São Vicente do Sul & Marchiori e Santos, 2009 \\
& 5916 & Barra do Quaraí & Marchiori e Santos, 2010b \\
Eugenia myrcianthes Nied. & 6078 & Nova Roma do Sul & Marchiori e Santos, 2010a \\
Eugenia rostrifolia D. Legrand & 6146 & Frederico Westphalen & Santos e Marchiori, 2011a \\
Eugenia schuechiana O. Berg & 2396 & São Vicente do Sul & Santos e Marchiori, 2009 \\
Eugenia uniflora Linnaus & 5910 & Santa Maria & Santos e Marchiori, 2009 \\
Eugenia uruguayensis Cambess. & 5808 & Rio Grande & Marchiori e Santos, 2010a \\
\hline
\end{tabular}

Em que: ${ }^{1}$ Registro em Xiloteca e Herbário do Departamento de Ciências Florestais da Universidade Federal de Santa Maria (HDCF). 
TABELA 2: Principais características anatômicas das 9 espécies investigadas.

TABLE 2: Main anatomical features of the 9 investigated species.

\begin{tabular}{|c|c|c|c|c|c|c|c|c|c|}
\hline Caráter anatômico & E.b. & E.h. & E.i. & E.ma. & E.my. & E.r. & E.s. & E.un. & E.ur. \\
\hline Poros exclusivamente solitários & + & + & + & + & + & - & + & + & + \\
\hline Curtos múltiplos radiais presentes & - & - & - & - & - & + & - & - & - \\
\hline Placas de perfuração simples & + & + & + & + & + & + & + & + & + \\
\hline Espessamentos espiralados & - & - & + & - & - & - & - & - & - \\
\hline Pontoações intervasculares alternas & + & + & + & + & + & + & + & + & + \\
\hline Pontoações raio-vasculares s.i.v. & + & + & + & + & + & + & + & + & + \\
\hline Parênquima difuso e difuso em agregados & + & + & + & + & + & + & + & + & + \\
\hline P. em faixas tangencias $>3$ células largura & - & - & - & - & + & + & - & + & - \\
\hline P. em linhas estreitas e descontínuas & - & + & - & + & - & - & - & - & - \\
\hline Séries cristalíferas no P. axial & + & + & + & + & + & + & + & + & + \\
\hline Raios heterogêneos & + & + & + & + & + & + & + & + & + \\
\hline Margens de até 4 células de altura & - & + & + & - & + & + & + & - & + \\
\hline Margens com mais de 4 células & + & - & - & + & - & - & - & + & - \\
\hline Fibras com pontoações areoladas & + & + & + & + & + & + & + & + & + \\
\hline Fibras de paredes espessas e/ou muito esp. & + & + & + & + & + & + & + & + & + \\
\hline Traqueídeos vasicêntricos & + & + & + & + & + & + & + & + & + \\
\hline Frequência de poros $\left(\mathrm{mm}^{2}\right)$ & 46 & 26 & 21 & 143 & 52 & 134 & 20 & 86 & 50 \\
\hline$\varnothing$ de poros $(\mu \mathrm{m})$ & 25 & 61 & 58 & 30 & 52 & 42 & 51 & 37 & 48 \\
\hline Comprimento de vasos $(\mu \mathrm{m})$ & 565 & 448 & 582 & 592 & 526 & 514 & 690 & 486 & 621 \\
\hline$\varnothing$ pontoações intervasculares $(\mu \mathrm{m})$ & 4,7 & 4,8 & 5,4 & 4,4 & 5,4 & 4,2 & 5,5 & 4,7 & 4,7 \\
\hline$\varnothing$ pontoações raio-vasculares $(\mu \mathrm{m})$ & 3,7 & 3,1 & 3,2 & 3,7 & 3 & 2,9 & 3,1 & 3,2 & 3,2 \\
\hline Altura séries P. axial (células) & $4-9$ & $2-10$ & $4-9$ & $4-11$ & $4-14$ & $4-13$ & $4-10$ & $3-9$ & $2-8$ \\
\hline Altura séries P. axial $(\mu \mathrm{m})$ & 547 & 462 & 583 & 581 & 550 & 547 & 710 & 402 & 538 \\
\hline Frequência de raios (mm) & 26 & 19 & 16 & 23 & 19 & 20 & 18 & 20 & 19 \\
\hline Largura de raios (células) & 2 & 3 & 2 & 2 & 2 & 3 & 2 & 2 & 3 \\
\hline Altura máx. de raios multiss. (células) & 26 & 17 & 18 & 24 & 15 & 21 & 38 & 20 & 19 \\
\hline Altura de raios multiss. $(\mu \mathrm{m})$ & 376 & 281 & 275 & 365 & 261 & 260 & 465 & 265 & 363 \\
\hline Altura máx. de raios uniss. (células) & 15 & 7 & 10 & 14 & 8 & 12 & 7 & 13 & 6 \\
\hline Altura de raios uniss. $(\mu \mathrm{m})$ & 216 & 184 & 168 & 257 & 167 & 172 & 258 & 162 & 205 \\
\hline Comprimento de fibras $(\mu \mathrm{m})$ & 1077 & 1086 & 1226 & 990 & 1154 & 995 & 1495 & 888 & 1236 \\
\hline
\end{tabular}

Em que: E.b. $=$ Eugenia burkartiana, E.h. $=$ Eugenia hiemalis, E.i. $=$ Eugenia involucrata, E.ma. $=$ Eugenia mansoi, E.my. $=$ Eugenia myrcianthes, E.r. $=$ Eugenia rostrifolia, E.s. $=$ Eugenia schuechiana, E.un. $=$ Eugenia uniflora, E.ur. $=$ Eugenia uruguayensis,$+=$ caráter presente, $-=$ caráter ausente, s.i.v $=$ semelhante às intervasculares, $\varnothing=$ diâmetro, $\mu \mathrm{m}=$ micrômetro, $\mathrm{mm}=$ milímetro, $\mathrm{P} .=$ parênquima, máx. $=$ máxima. Os números correspondem aos valores médios, exceto quando especificado o contrário.

rio-grandenses de Eugenia (Tabela 1). Os dados qualitativos e quantitativos da estrutura anatômica das espécies (Tabela 2), base do presente estudo, foram colhidos de publicações anteriores, elaboradas a partir de material coletado no Rio Grande do Sul (Tabela 1). Reunidos os dados, procedeu-se a comparação dos mesmos entre si e com referências da literatura, buscando-se identificar caracteres de valor diagnóstico para o gênero e espécies.

As fotomicrografias da estrutura anatômica foram tomadas, a partir de lâminas permanentes coloridas artificialmente, em microscópio Olympus CX40, equipado com câmera digital Olympus Camedia c3000, no Laboratório de Anatomia da Madeira da Universidade Federal do Paraná. Maiores detalhes sobre o preparo das lâminas 
e coleta das amostras podem ser obtidos nas publicações originais, listadas nas referências.

\section{RESULTADOS E DISCUSSÃO}

As características anatômicas da madeira das nove espécies em estudo estão dentro das possibilidades estruturais para a família Myrtaceae (RECORD e HESS, 1949; VLIET e BAAS, 1984) e gênero Eugenia (METCALFE e CHALK, 1972). Verificou-se uma grande homogeneidade estrutural entre as espécies investigadas, reflexo do elevado número de caracteres anatômicos compartilhados: porosidade difusa; elementos vasculares de comprimento médio; placas de perfuração simples; pontoações intervasculares alternas, pequenas e ornamentadas; pontoações raio-vasculares semelhantes às intervasculares, restritas às margens; parênquima axial apotraqueal difuso e difuso em agregados, em séries de 4-8 células (altura predominante); raios heterogêneos estreitos, com células de paredes disjuntas e menos de $1 \mathrm{~mm}$ de altura; fibras com pontoações areoladas, de paredes espessas e/ou muito espessas; traqueídeos vasicêntricos; e cristais. Este conjunto de características é também compartilhado pelos demais representantes de Eugenia referidos na literatura, bem como com a maioria das Myrtoídeas (BARROS e CALADO, 1997; SOFFIATTI e ANGYALOSSY-ALFONSO, 1999; BARROS et al., 2001, 2003). Exceções, contudo, ocorrem, como a presença de fibras libriformes, citadas para certas espécies de Eugenia por Metcalfe e Chalk (1972) e Rebollar-Domínguez e Tapia-Torres (2010).

Détienne e Jacquet (1983) e Soffiatti e Angyalossy-Alfonso (1999) observaram, igualmente, uma grande homogeneidade estrutural no gênero em estudo. Record e Hess (1949), por sua vez, salientam que a variabilidade nas madeiras de Eugenia é tão grande quanto a observada entre os diferentes gêneros da subfamília Myrtoideae. Isso, no entanto, não contraria os resultados presentemente obtidos, bem como o referido por Détienne e Jacquet (1983) e Soffiatti e AngyalossyAlfonso (1999), posto que a diversidade estrutural nesta subfamília é, também, relativamente pequena, como demonstra o trabalho de Dias-Leme et al., (1995).

As espécies estudadas diferem principalmente no tocante ao arranjo do parênquima axial, frequência e diâmetro de poros, e altura de raios (Tabela 2).
Todas as espécies apresentam parênquima apotraqueal difuso e difuso em agregados. Estes padrões, todavia, nem sempre são predominantes na madeira: Eugenia myrcianthes, Eugenia rostrifolia e Eugenia uniflora têm parênquima em faixas com mais de três células de largura; Eugenia hiemalis e E. mansoi apresentam parênquima principalmente em linhas estreitas e descontínuas (Figura 1).

$\mathrm{O}$ arranjo do parênquima axial tem sido considerado o caráter de maior importância para a separação de espécies na família (DIAS-LEME et al., 1995), inclusive em Eugenia (DÉTIENNE e JACQUET, 1983; SOFFIATTI e ANGYALOSSYALFONSO, 1999). Os resultados do presente estudo contribuem positivamente com este posicionamento, tendo em vista que o arranjo do parênquima axial é a característica (qualitativa) de maior variabilidade entre as madeiras.

Das características quantitativas em destaque, a frequência de poros foi a que apresentou a maior variabilidade, sendo, por este motivo, especialmente importante sob o ponto de vista taxonômico. Como se pode observar na Tabela 2, há espécies com menos de 30 poros $/ \mathrm{mm}^{2}$ (Eugenia involucrata, Eugenia schuechiana e Eugenia hiemalis), até mais de 100 poros $/ \mathrm{mm}^{2}$ (Eugenia mansoi e Eugenia rostrifolia).

As características quantitativas das espécies em estudo, especialmente frequência e diâmetro de poros e comprimento de vasos, diferem na comparação com a maioria das espécies de Eugenia de latitudes menores (BARROS et al., 2001, 2003): o diâmetro e o comprimento de vasos tendem a ser maiores e a frequência de poros menor que a observada. Este fato deve ser levado em conta ao se comparar espécies de regiões distintas. O mesmo raciocínio vale para espécies de ambientes com diferenças na disponibilidade hídrica, como demonstrado em inúmeros trabalhos de anatomia ecológica (BAAS et al., 1983; LINDORF, 1994; DENARDI e MARCHIORI, 2005).

A altura das séries de parênquima axial, comprimento de vasos e fibras, bem como o diâmetro de pontoações intervasculares e raio-vasculares não são bons critérios, devido à baixa variabilidade apresentada, servindo apenas ocasionalmente para a separação de espécies. A largura de raios, assim como o comprimento das margens unisseriadas, por outro lado, são aspectos bastante úteis para este fim, apesar de pouco variáveis (Tabela 2; Figura 2).

Digno de nota é a presença de séries cristalíferas no parênquima axial. Todas as espécies 

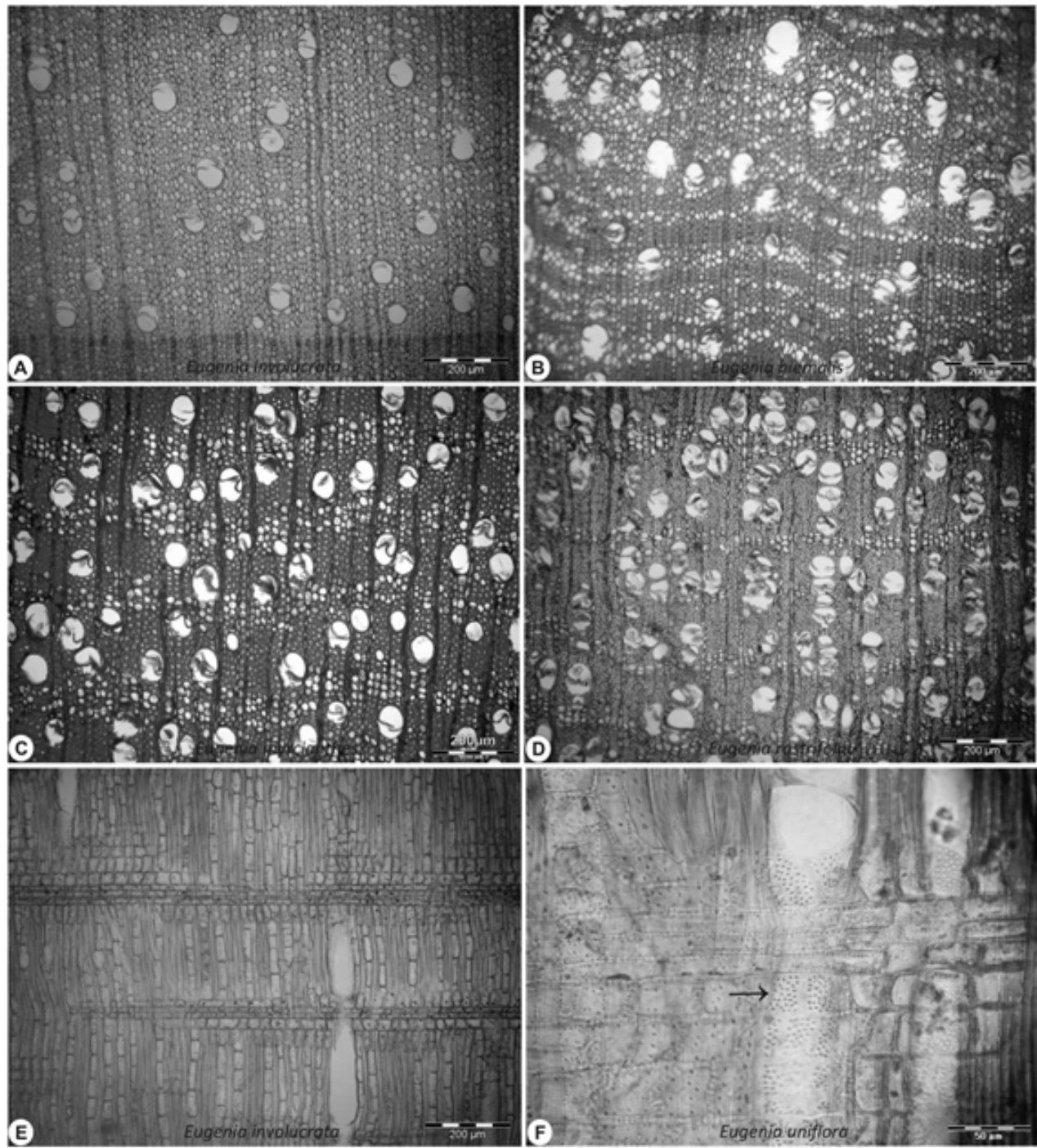

FIGURA 1: Aspectos anatômicos em madeiras de Eugenia. A-D. Diversidade no arranjo do parênquima axial, em seção transversal: parênquima apotraqueal difuso e difuso em agregados (A); parênquima em linhas descontínuas e estreitas (B); parênquima em faixas contínuas, com mais de três células de largura (C,D). Notar, também, a presença de vasos em curtos múltiplos radiais (D). E. Raio heterogêneo, com células procumbentes (corpo central), quadradas e eretas (margens), em seção longitudinal radial. F. Mesma seção da foto anterior, mostrando pontoações raio-vasculares semelhantes às intervasculares, restritas às margens (seta).

FIGURE 1: Wood anatomical features in Eugenia.

de Eugenia investigadas contam com este caráter anatômico (Tabela 2), a exemplo dos demais representantes brasileiros do gênero, referidos na literatura. Os cristais sempre ocorrem em idioblastos, com exceção de Eugenia involucrata e Eugenia myrcianthes, que têm cristais em câmaras de tamanho semelhantes às células de parênquima normais (Figura 2). Cabe salientar que Barros et al. (2003) não fazem menção à ocorrência ou não de cristais na estrutura anatômica de Eugenia hiemalis; na amostra de madeira presentemente estudada desta espécie, todavia, o caráter é observado.

A ocorrência de cristais não é exclusiva do gênero Eugenia (METCALFE e CHALK, 1972; VLIET e BAAS, 1984; DIAS-LEME et al., 1995). Nem todos os gêneros da família, por outro lado, contam com este detalhe anatômico. Em Campomanesia, por exemplo, não há registro da presença de cristais. O padrão de distribuição constante, verificado em Eugenia, recomenda o 


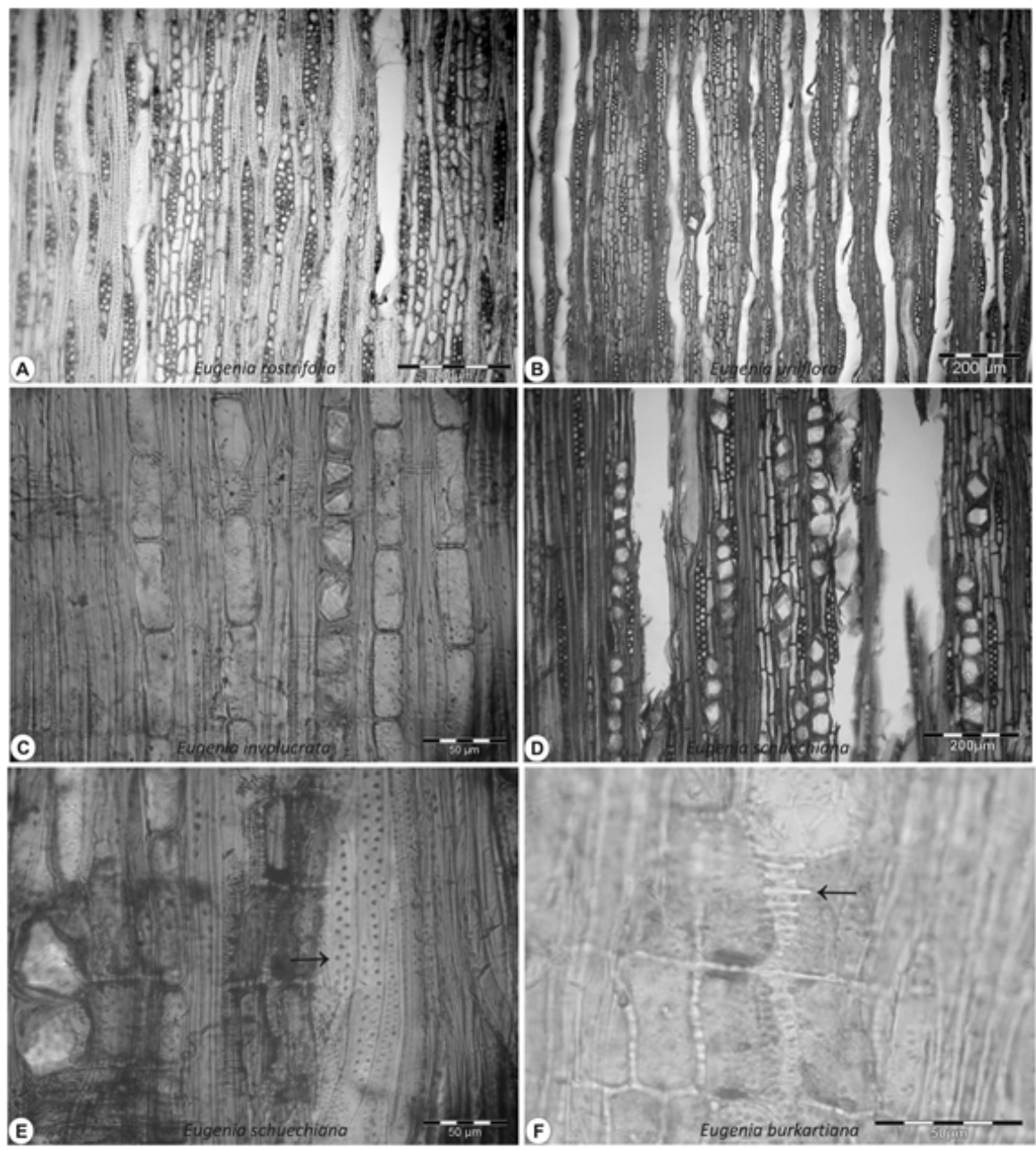

FIGURA 2: Aspectos anatômicos em madeiras de Eugenia. A. Raios com 1-3 células de largura e margens unisseriadas de até 4 células (seção longitudinal tangencial). B. Raios com 1-2 células de largura e margens unisseriadas geralmente com mais de 4 células (seção longitudinal tangencial). C. Seção longitudinal radial, mostrando séries cristalíferas em câmaras não distendidas, no parênquima axial. D. Cristais em idioblastos, no parênquima axial (seção longitudinal tangencial). E. Traqueídeos vasicêntricos (seta), em vista longitudinal radial. F. Mesmo plano anatômico da foto anterior, com destaque para células radiais de paredes disjuntas (seta).

FIGURE 2: Wood anatomical features in species of Eugenia.

emprego deste caráter para fins taxonômicos, na ausência de aspectos diferenciais mais importantes, ainda mais se considerada a grande homogeneidade estrutural da família. Dias-Leme et al. (1995) e Soffiatti e Angyalossy-Alfonso (1999) são alguns dos autores que se valeram deste critério para a separação de espécies em Myrtaceae. Cury (2001) refere a presença de cristais nos raios de Eugenia uniflora. Ao contrário do observado por este autor, não foram encontrados cristais nos raios da pitangueira, bem como dos demais representantes do gênero incluídos no presente estudo. Sobre a ocorrência de cristais na madeira, vale lembrar que o caráter pode ter natureza ocasional em alguns grupos (WHEELER et al., 1989), motivo pelo qual nem todos defendem o emprego do mesmo para a identificação de espécies (CORE et al., 1979).

Merece comentário, também, as ornamentações no pontoado intervascular, caráter considerado conservativo para a família Myrtaceae e ordem Myrtales como um todo (JANSEN et al., 2001, 2008). Contrariando a tendência geral, não foram observadas pontoações ornamentadas na madeira de Eugenia uniflora e Eugenia uruguayensis. A ausência de ornamentações em Eugenia uniflora não é corroborada por Soffiatti e 
Angyalossy-Alfonso (1999). Vale lembrar que nem sempre é possível observar ornamentações através da microscopia óptica, mesmo nos grupos em que o caráter sabidamente ocorre. Como explicam Wheeler et al. (1989), o grau de desenvolvimento, bem como a forma e distribuição das ornamentações, variam de acordo com o grupo taxonômico. Quando as pontoações são grandes e as ornamentações bem desenvolvidas, estas são relativamente fáceis de serem vistas em microscopia convencional, caso contrário, a visualização torna-se difícil. A não observação de ornamentações nas espécies em estudo, portanto, pode dever-se tão simplesmente ao reduzido desenvolvimento das mesmas, do que propriamente a sua ausência.

Nenhuma característica anatômica ocorre de maneira exclusiva no gênero em estudo, impossibilitando sua identificação através da anatomia da madeira. Em trabalho sobre a subtribo Myrciinae, Dias-Leme et al. (1995) também não conseguiram separar os gêneros com base na anatomia da madeira, devido à falta de características exclusivas para os mesmos. Aseparação das espécies, entretanto, é possível de ser realizada, especialmente quando a madeira exibe características anatômicas de ocorrência mais restrita, caso da presença de espessamentos espiralados (Eugenia involucrata) e de poros em múltiplos radiais (Eugenia rostrifolia), por exemplo.

As características anatômicas observadas em Hexachlamys edulis (Eugenia myrcianthes) incluem-se nas possibilidades estruturais de Eugenia, motivo pelo qual não é possível contestar, com base na anatomia da madeira, a sua inclusão neste gênero, como sugerido por Landrum e Kawasaki (1997). Além disso, como já salientado, faltam características anatômicas específicas para os gêneros em Myrtaceae, incluindo Hexachlamys e Eugenia, o que limita a atuação da anatomia da madeira em discussões taxonômicas.

\section{CONCLUSÕES}

As características anatômicas da madeira das nove espécies em estudo estão dentro das possibilidades estruturais referidas na literatura para a família Myrtaceae e gênero Eugenia;

Há uma grande homogeneidade estrutural no referido gênero, fruto do elevado número de caracteres anatômicos compartilhados entre as espécies;

Nenhuma característica anatômica pode ser reconhecida como exclusiva de Eugenia, motivo pelo qual não é possível identificar o gênero através da anatomia da madeira;

É confirmado o valor do arranjo do parênquima axial para a separação de espécies, sendo também importantes, neste sentido, a frequência de poros e certas características de raios;

Não se pode contestar, com base na anatomia da madeira, a inclusão de Hexachlamys em Eugenia, como sugerido por Landrum e Kawasaki (1997).

\section{AGRADECIMENTOS}

Os autores agradecem ao Laboratório de Anatomia da Madeira da Universidade Federal do Paraná, onde foram obtidas as fotomicrografias das madeiras, e ao $\mathrm{CNPq}$, pelo auxílio financeiro.

\section{REFERÊNCIAS BIBLIOGRÁFICAS}

\section{BARROS, C. F. et al. Madeiras da Mata Atlântica.} Anatomia do lenho de espécies ocorrentes nos remanescentes florestais do estado do Rio de Janeiro, Brasil. Rio de Janeiro: Instituto de Pesquisas Jardim Botânico do Rio de Janeiro. 2001. 94 p.

BARROS, C. F. et al. Madeiras da Mata Atlântica. Anatomia do lenho de espécies ocorrentes nos remanescentes florestais do estado do Rio de Janeiro, Brasil. Rio de Janeiro: Instituto de Pesquisas Jardim Botânico do Rio de Janeiro. 2003. 86 p.

BARROSO, G. M. Sistemática de Angiospermas do Brasil. Viçosa: UFV, 1991. 377 p.

CORE, H. A.; CÔTÉ, W. A.; DAY, A. C. Wood structure and identification. New York: Syracuse University Press, 1979. 182 p.

BAAS, P.; WERKER, E.; FAHN, A. Some ecological trends in vessel characters. IAWA Bulletin, v. 4, p. 141-160, 1983.

CURY, G. Descrição da estrutura anatômica do lenho e sua aplicação na identificação de espécies arbóreas do cerrado e da mata atlântica do estado de São Paulo. 2001. 125 f. Dissertação (Mestrado) - Universidade de São Paulo: Escola Superior de Agricultura Luiz de Queiroz, Piracicaba, 2001.

DENARDI, L.; MARCHIORI, J. N. C. Anatomia ecológica da madeira de Blepharocalyx salicifolius (H. B. K.) Berg. Ciência Florestal, Santa Maria, v. 15, n. 2, p. 119-127, 2005.

DÉTIENNE,P.; JACQUET,P. Atlas d'identification des bois de l'Amazonie et des regions voisines. Centre Technique Forestier Tropical, Nogent-surMarne, 1983. 
DIAS-LEME, C. L.; GASSON, P.; LUGHADA, E. N. Wood anatomy of four Myrtaceae genera in the subtribe Myciinae from South America. IAWA Bulletin, n. 16, p. 87-95, 1995.

JANSEN, S; BAAS, P; SMETS, E. Vestured pits: their occurrence and systematic importance in eudicots. Taxon, v. 50, n. 1, p. 135-167, 2001.

JANSEN, S. et al. Vestured pits: a diagnostic character in the secondary xylem of Myrtales. Journal of Tropical Forest Science, v. 20, n. 4, p. 328-339, 2008.

KLEIN, R. M. Importância sociológica das Mirtáceas nas florestas rio-grandenses. In: CONGRESSO NACIONAL DE BOTÂNICA, 34., 1984, Porto Alegre. Anais... Porto Alegre, 1984. p. 367-375.

LANDRUM, L. R.; KAWASAKI, M. L. The genera of Myrtaceae in Brasil: an illustrated synoptic treatment and identification keys. Brittonia, n. 49, p. 508-536, 1997.

LINDORF, H. Eco-anatomical wood features of species from a very dry tropical forest. IAWA Bulletin, v. 15, p. 361-376, 1994.

MAINIERI, C.; CHIMELO, J. P. Fichas de características das madeiras brasileiras. São Paulo: Instituto de Pesquisa Tecnológicas, Divisão de Madeiras, 1989. 418 p.

MARCHIORI, J. N. C. Anatomia da madeira de Eugenia involucrata DC. (Myrtaceae). Ciência e Natura, Santa Maria, v. 6, p. 127-136, 1984.

MARCHIORI, J. N. C.; SANTOS, S. R. Anatomia do xilema secundário de Eugenia mansoi O. Berg (Myrtaceae). Balduinia, Santa Maria, n. 16, p. 6-12, 2009.

MARCHIORI, J. N. C.; SANTOS, S. R. Anatomia da madeira de duas espécies de Eugenia L. (Myrtaceae). Balduinia, n. 21, p. 15-21, 2010a.

MARCHIORI, J. N. C.; SANTOS, S. R. Anatomia das madeiras de Campomanesia aurea O. Berg e Eugenia myrcianthes Niedenzu (Myrtaceae). Balduinia, Santa Maria, n. 22, p. 23-30, 2010b. MARCHIORI, J. N. C.; SOBRAL, M. Dendrologia das Angiospermas. Myrtales. Santa Maria: Ed. da UFSM, 1997. $304 \mathrm{p}$.

MARQUES, P. A. et al. Anatomia do lenho de três espécies de Eugenia L. (Myrtaceae) de mata e restinga. Revista Brasileira de Biociências, v. 5, p. 801-803, 2007.

MERWE, M. M. van der; WYK, A. E. van; BOTHA, A. M. Molecular phylogenetic analysis of Eugenia L. (Myrtaceae), with emphasis on southern African taxa. Plant Systematics and Evolution, v. 251, p. 21-34, 2005.

METCALFE, C. R.; CHALK, L. Anatomy of the Dicotyledons. Oxford: Clarendon Press, 1972. $1500 \mathrm{p}$.

METCALFE, C. R.; CHALK, L. Anatomy of the dicotyledons. Wood structure and conclusions of the general introdution. Oxford: Clarendon Press, 1983.

OLIVEIRA, F. Aspectos da vegetação arbórea encontrada na orla da Praia da Alegria no município de Guaíba, RS, Brasil. Caderno de Pesquisa, série Biológica, v. 19, p. 6-17, 2007.

REBOLLAR-DOMÍNGUEZ, S.; TAPIA-TORRES, N.A. Anatomía de la madera de dos especies de Eugenia (Myrtaceae) de Quintana Roo, México. Madera y Bosques, v. 16, n. 1, p. 85-98, 2010.

RECORD, S. J.; HESS, R.W. Timbers of the New World. New Haven: Yale University Press, 1949. $640 \mathrm{p}$.

SANTOS, S. R.; MARCHIORI, J. N. C. Anatomia da madeira de Eugenia uniflora L. (Myrtaceae). Balduinia, Santa Maria, v. 17, p. 11-16, 2009.

SANTOS, S. R.; MARCHIORI, J. N. C. Anatomia das madeiras de Eugenia burkartiana (D. Legrand) D. Legrand e Myrciaria cuspidata O. Berg, duas Myrtoídeas nativas no Rio Grande do Sul. Balduinia, Santa Maria, v. 22, p. 15-22, 2010.

SANTOS, S. R.; MARCHIORI, J. N. C. Estudo anatômico do lenho de Eugenia schuechiana O. Berg (Myrtaceae). Balduinia, Santa Maria, v. 26, p. 27-32, 2011a.

SANTOS, S. R.; MARCHIORI, J. N. C. Estudo anatômico do lenho de Eugenia hiemalis Cambess. (Myrtaceae). Balduinia, Santa Maria, v. 31 p. 27-32, 2011b.

SOBRAL, M. A família Myrtaceae no Rio Grande do Sul. São Leopoldo: Unisinos, 2003. 215 p.

SOFFIATTI, P.; ALFONSO, V.A. Estudo anatômico comparativo do lenho e da casca de duas espécies de Eugenia L. (Myrtaceae). Revista Brasileira de Botânica, São Paulo, v. 22, n. 2, p. 175-184, 1999. VLIET, G. J. C. van; BAAS, P. Wood anatomy and classification of the Myrtales. Annals of the Missouri Botanical Garden, n. 71, p. 783-800, 1984.

WHEELER, E. A.; BAAS, P.; GASSON, P. E. IAWA list of microscopic features for hardwood identification. IAWA Bulletin, v.10, n. 3, p. 218-359, 1989. 